Acta Crystallographica Section E

Structure Reports

Online

ISSN 1600-5368

\section{Indomethacin methyl ester}

\section{Andrew V. Trask, ${ }^{a *}$ Ning Shan, ${ }^{b}$ William Jones ${ }^{\mathrm{a}}$ and W. D. Sam Motherwell $^{\mathrm{c}}$}

${ }^{a}$ Department of Chemistry, University of Cambridge, Lensfield Road, Cambridge CB2 1EW, England, and Pfizer Institute for Pharmaceutical Materials Science, ${ }^{\mathbf{b}}$ Institute of Chemical and Engineering Sciences, Ayer Rajah Crescent, Block 28, Unit 02-08, Singapore 139959, and Pfizer Institute for Pharmaceutical Materials Science, and ${ }^{\mathbf{c}}$ Cambridge Crystallographic Data Centre, 12 Union Road, Cambridge CB2 1EZ, England

Correspondence e-mail: avt21@cam.ac.uk

\section{Key indicators}

Single-crystal X-ray study

$T=180 \mathrm{~K}$

Mean $\sigma(\mathrm{C}-\mathrm{C})=0.003 \AA$

$R$ factor $=0.047$

$w R$ factor $=0.117$

Data-to-parameter ratio $=13.0$

For details of how these key indicators were automatically derived from the article, see http://journals.iucr.org/e.
The cystal structure of the title compound [systematic name: methyl 1-(4-chlorobenzoyl)-5-methoxy-2-methyl- $1 H$-indole-3acetate], $\mathrm{C}_{20} \mathrm{H}_{18} \mathrm{ClNO}_{4}$, exhibits a short axis similar to another indomethacin analogue. Also observed in the structure is a packing of molecules influenced by weak $\mathrm{C}-\mathrm{H} \cdots \mathrm{O}$ hydrogen bonds.

\section{Comment}

As part of an investigation into the crystallization of pharmaceutical compounds, the crystal structures of indomethacin derivatives are of interest. Numerous studies have been reported on various crystal structures of the drug indomethacin ( $\gamma$-form: Kistenmacher \& Marsh, 1972; $\alpha$-form: Chen et al., 2002; $t$-butanol and methanol solvates: Joshi et al., 1998). In contrast, the structure of its methyl ester, (I), has not been reported to date. We report here its crystal structure and describe the intermolecular interactions involved.

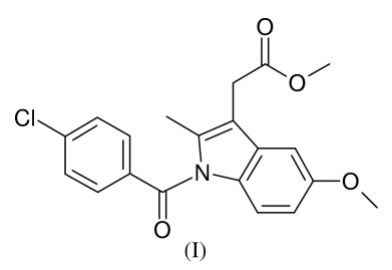

The asymmetric unit of (I) comprises one molecule (Fig. 1). Although the crystal structure of the indomethacin methyl ester differs significantly from that of the parent carboxylic acid, it bears some similarity to the structure of another indomethacin derivative, iodoindomethacin (Loll et al., 1996). Both crystal structures exhibit a relatively short axis [4.8326 (1) A for the methyl ester derivative versus 4.7250 (10) $\AA$ for the iodo derivative]. In addition, both crystal structures show a halogen contact to a carbonyl $\mathrm{O}$ atom $[\mathrm{Cl} 1 \cdots \mathrm{O} 4=$ 3.575 (2) $\AA$ versus $\mathrm{I} 1 \cdots \mathrm{O} 4=3.162(5) \AA]$.

In the absence of the carboxylic acid group of indomethacin, no strong $\mathrm{O}-\mathrm{H} \cdots \mathrm{O}$ hydrogen bonding can be expected in the crystal structure of the methyl ester. Instead, $\mathrm{C}-\mathrm{H} \cdots \mathrm{O}$ hydrogen bonds form a three-dimensional supramolecular network, as shown in Fig. 2. Hydrogen-bond distances and angles are provided in Table 1.

\section{Experimental}

Indomethacin and anhydrous benzenesulfonic acid were obtained from Sigma-Aldrich and were used as received. Indomethacin (130 mg, $0.364 \mathrm{mmol})$ and benzensulfonic acid $(115 \mathrm{mg}, 0.727 \mathrm{mmol})$ were dissolved in methanol with heating. Crystals precipitated as the
Received 30 January 2004 Accepted 26 February 2004 Online 6 March 2004 


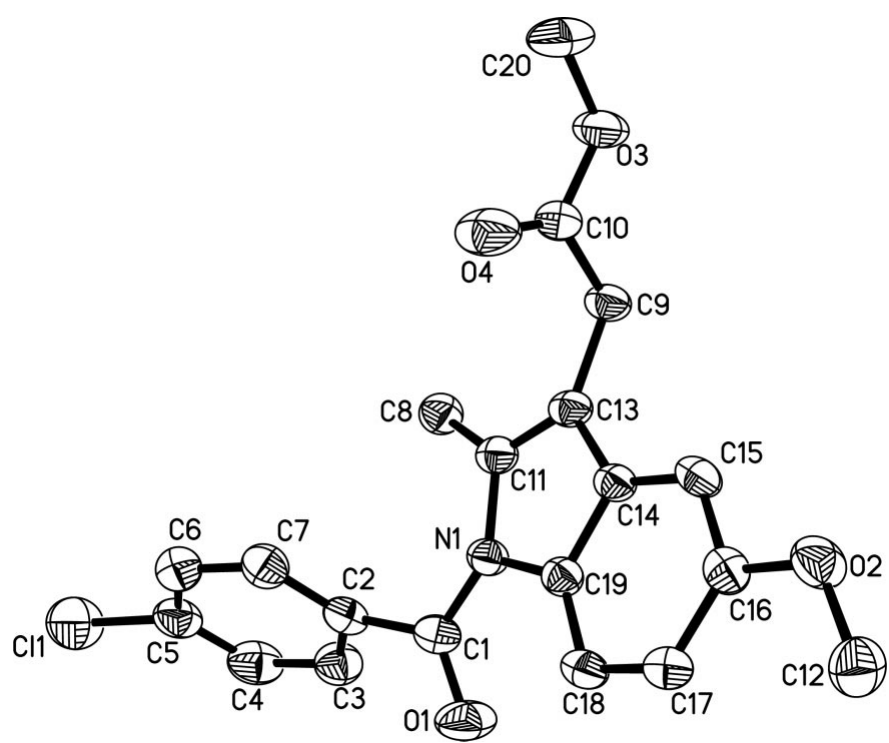

Figure 1

Molecular unit showing displacement ellipsoids at the $50 \%$ probability level.

solution cooled to room temperature and were immediately isolated and dried.

$$
\begin{aligned}
& \text { Crystal data } \\
& \mathrm{C}_{20} \mathrm{H}_{18} \mathrm{ClNO}_{4} \\
& M_{r}=371.82 \\
& \text { Monoclinic, } P 2_{1} / n \\
& a=19.0206(5) \AA \\
& b=4.8326(1) \AA \\
& c=19.3092(8) \AA \\
& \beta=97.739(1)^{\circ} \\
& V=1758.72(9) \AA^{3} \\
& Z=4
\end{aligned}
$$

Data collection

Nonius KappaCCD diffractometer $\omega$ and $\varphi$ scans

Absorption correction: multi-scan

(SORTAV; Blessing, 1995)

$T_{\min }=0.946, T_{\max }=0.985$

10009 measured reflections

3086 independent reflections

\section{Refinement}

Refinement on $F^{2}$

$R\left[F^{2}>2 \sigma\left(F^{2}\right)\right]=0.047$

$w R\left(F^{2}\right)=0.117$

$S=1.04$

3086 reflections

238 parameters

$\mathrm{H}$-atom parameters constrained

\author{
$D_{x}=1.404 \mathrm{Mg} \mathrm{m}^{-3}$ \\ Mo $K \alpha$ radiation \\ Cell parameters from 6724 \\ reflections \\ $\theta=1.0-25.0^{\circ}$ \\ $\mu=0.24 \mathrm{~mm}^{-1}$ \\ $T=180(2) \mathrm{K}$ \\ Needle, yellow \\ $0.46 \times 0.07 \times 0.05 \mathrm{~mm}$
}

$$
\begin{aligned}
& 2410 \text { reflections with } I>2 \sigma(I) \\
& R_{\text {int }}=0.035 \\
& \theta_{\max }=25.0^{\circ} \\
& h=-22 \rightarrow 22 \\
& k=-5 \rightarrow 5 \\
& l=-22 \rightarrow 23
\end{aligned}
$$

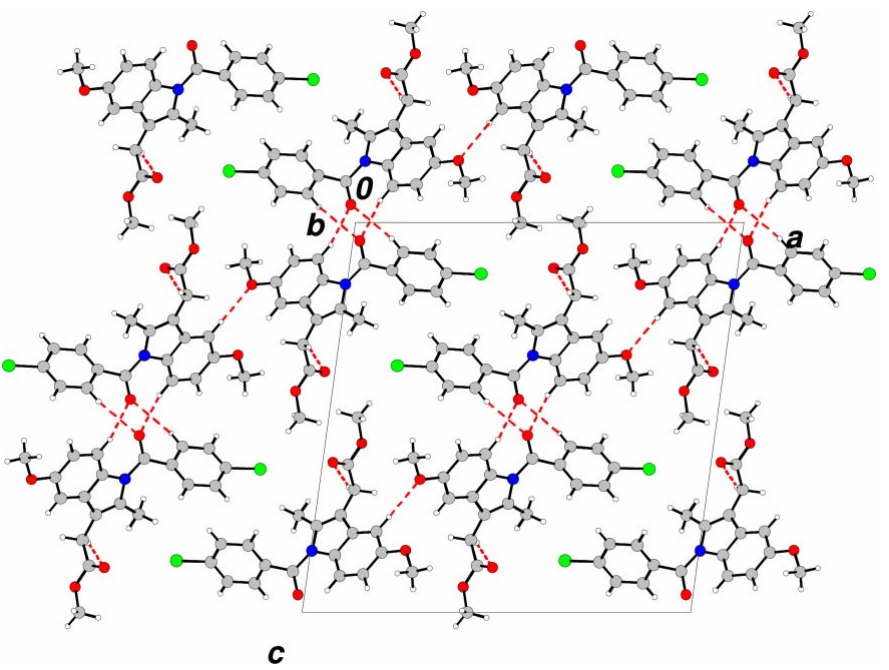

Figure 2

Projection on to (010), showing the packing involving $\mathrm{C}-\mathrm{H} \cdots \mathrm{O}$ interactions. The intermolecular $\mathrm{C} 9-\mathrm{H} 9 A \cdots \mathrm{O} 4(x, y-1, z)$ hydrogen bond projects parallel to the $b$ axis.

All $\mathrm{H}$ atoms were placed geometrically and treated using a riding model. The $U_{\text {iso }}$ values for methyl $\mathrm{H}$ atoms were fixed at $1.5 U_{\text {eq }}$ of the carrier atom. For all other $\mathrm{H}$ atoms, $U_{\text {iso }}(\mathrm{H})=1.2 U_{\text {eq }}$ (carrier atom). The $\mathrm{C}-\mathrm{H}$ distances of methyl groups were fixed at $0.98 \AA$; all other $\mathrm{C}-\mathrm{H}$ distances were fixed at $0.95 \AA$.

Data collection: COLLECT (Nonius, 1998); cell refinement: $H K L$ SCALEPACK (Otwinowski \& Minor, 1997); data reduction: $H K L$ DENZO (Otwinowski \& Minor, 1997) and SCALEPACK; program(s) used to solve structure: SHELXS97 (Sheldrick, 1997); program(s) used to refine structure: SHELXL97 (Sheldrick, 1997); molecular graphics: $X P$ (Sheldrick, 1993) and DIAMOND (Brandenburg, 1999); software used to prepare material for publication: SHELXL97.

The authors are grateful for funding from the Pfizer Institute for Pharmaceutical Materials Science at the University of Cambridge. We thank Dr J. E. Davies for the data collection.

\section{References}

Blessing, R. H. (1995). Acta Cryst. A51, 33-38.

Brandenburg, K. (1999). DIAMOND. Version 2.1c. Crystal Impact GbR, Bonn, Germany.

Chen, X., Morris, K. R., Griesser, U. J., Byrn, S. R. \& Stowell, J. G. (2002). J. Am. Chem. Soc. 124, 15012-15019.

Joshi, V., Stowell, J. G. \& Byrn, S. R. (1998). Mol. Cryst. Liq. Cryst. 313, 265270.

Kistenmacher, T. J. \& Marsh, R. E. (1972). J. Am. Chem. Soc. 94, 1340-1345. Loll, P. J., Garavito, R. M., Carrell, C. J. \& Carrell, H. L. (1996). Acta Cryst. C52, 455-457.

Nonius (1998). COLLECT. Nonius BV, Delft, The Netherlands.

Otwinowski, Z. \& Minor, W. (1997). Methods in Enzymology, Vol. 276,

Macromolecular Crystallography, Part A, edited by C. W. Carter Jr \& R. M.

Sweet, pp. 307-326. New York: Academic Press.

Sheldrick, G. M. (1993). XP. University of Göttingen, Germany.

Sheldrick, G. M. (1997). SHELXL97 and SHELXS97. University of Göttingen, Germany. 


\section{supporting information}

Acta Cryst. (2004). E60, o508-o509 [https://doi.org/10.1107/S1600536804004453]

\section{Indomethacin methyl ester}

\section{Andrew V. Trask, Ning Shan, William Jones and W. D. Sam Motherwell}

Methyl [1-(4-chlorobenzoyl)-5-methoxy-2-methyl-1H-indol-3-ylmethyl]acetate

Crystal data

$\mathrm{C}_{20} \mathrm{H}_{18} \mathrm{ClNO}_{4}$

$M_{r}=371.82$

Monoclinic, $P 2_{1} / n$

$a=19.0206(5) \AA$

$b=4.8326(1) \AA$

$c=19.3092(8) \AA$

$\beta=97.739(1)^{\circ}$

$V=1758.72(9) \AA^{3}$

$Z=4$

$F(000)=776$

\section{Data collection}

Nonius KappaCCD diffractometer

Radiation source: fine-focus sealed tube Graphite monochromator

$\omega$ and $\varphi$ scans

Absorption correction: multi-scan

Sortav (Blessing, 1995)

$T_{\min }=0.946, T_{\max }=0.985$

Refinement

Refinement on $F^{2}$

Least-squares matrix: full

$R\left[F^{2}>2 \sigma\left(F^{2}\right)\right]=0.047$

$w R\left(F^{2}\right)=0.117$

$S=1.04$

3086 reflections

238 parameters

0 restraints

Primary atom site location: structure-invariant direct methods
$D_{\mathrm{x}}=1.404 \mathrm{Mg} \mathrm{m}^{-3}$

Melting point: not measured $\mathrm{K}$

Mo $K \alpha$ radiation, $\lambda=0.71073 \AA$

Cell parameters from 6724 reflections

$\theta=1.0-25.0^{\circ}$

$\mu=0.24 \mathrm{~mm}^{-1}$

$T=180 \mathrm{~K}$

Needle, yellow

$0.46 \times 0.07 \times 0.05 \mathrm{~mm}$

10009 measured reflections

3086 independent reflections

2410 reflections with $I>2 \sigma(I)$

$R_{\text {int }}=0.035$

$\theta_{\max }=25.0^{\circ}, \theta_{\min }=3.6^{\circ}$

$h=-22 \rightarrow 22$

$k=-5 \rightarrow 5$

$l=-22 \rightarrow 23$

Secondary atom site location: difference Fourier map

Hydrogen site location: inferred from neighbouring sites

$\mathrm{H}$-atom parameters constrained

$w=1 /\left[\sigma^{2}\left(F_{\mathrm{o}}^{2}\right)+(0.0481 P)^{2}+1.0445 P\right]$

where $P=\left(F_{\mathrm{o}}^{2}+2 F_{\mathrm{c}}{ }^{2}\right) / 3$

$(\Delta / \sigma)_{\max }=0.002$

$\Delta \rho_{\max }=0.31 \mathrm{e} \AA^{-3}$

$\Delta \rho_{\min }=-0.39$ e $\AA^{-3}$

Special details

Geometry. All e.s.d.'s (except the e.s.d. in the dihedral angle between two 1.s. planes) are estimated using the full covariance matrix. The cell e.s.d.'s are taken into account individually in the estimation of e.s.d.'s in distances, angles and torsion angles; correlations between e.s.d.'s in cell parameters are only used when they are defined by crystal symmetry. An approximate (isotropic) treatment of cell e.s.d.'s is used for estimating e.s.d.'s involving 1.s. planes. 
Refinement. Refinement of $F^{2}$ against ALL reflections. The weighted $R$-factor $w R$ and goodness of fit $S$ are based on $F^{2}$, conventional $R$-factors $R$ are based on $F$, with $F$ set to zero for negative $F^{2}$. The threshold expression of $F^{2}>\sigma\left(F^{2}\right)$ is used only for calculating $R$-factors(gt) etc. and is not relevant to the choice of reflections for refinement. $R$-factors based on $F^{2}$ are statistically about twice as large as those based on $F$, and $R$ - factors based on ALL data will be even larger.

Fractional atomic coordinates and isotropic or equivalent isotropic displacement parameters $\left(\AA^{2}\right)$

\begin{tabular}{|c|c|c|c|c|}
\hline & $x$ & $y$ & $z$ & $U_{\text {iso }} * / U_{\text {eq }}$ \\
\hline $\mathrm{Cl1}$ & $0.65787(4)$ & $-0.17803(18)$ & $0.86754(4)$ & $0.0706(3)$ \\
\hline $\mathrm{O} 1$ & $0.98228(10)$ & $0.2360(4)$ & $0.95373(9)$ & $0.0550(5)$ \\
\hline $\mathrm{O} 2$ & $1.24396(8)$ & $0.8532(3)$ & $0.84051(8)$ & $0.0436(4)$ \\
\hline $\mathrm{O} 3$ & $1.09430(9)$ & $0.0986(3)$ & $0.56653(8)$ & $0.0458(4)$ \\
\hline $\mathrm{O} 4$ & $1.02616(11)$ & $0.3946(4)$ & $0.61588(9)$ & $0.0613(5)$ \\
\hline N1 & $1.00383(9)$ & $0.2127(3)$ & $0.84188(9)$ & $0.0299(4)$ \\
\hline $\mathrm{C} 1$ & $0.96165(12)$ & $0.1665(5)$ & $0.89405(11)$ & $0.0355(5)$ \\
\hline $\mathrm{C} 2$ & $0.88813(12)$ & $0.0526(5)$ & $0.87820(11)$ & $0.0354(5)$ \\
\hline $\mathrm{C} 3$ & $0.86722(13)$ & $-0.1409(5)$ & $0.92464(12)$ & $0.0431(6)$ \\
\hline H3 & 0.9013 & -0.2196 & 0.9596 & $0.052 *$ \\
\hline $\mathrm{C} 4$ & $0.79675(14)$ & $-0.2190(5)$ & $0.92002(13)$ & $0.0498(7)$ \\
\hline $\mathrm{H} 4$ & 0.7824 & -0.3554 & 0.9507 & $0.060 *$ \\
\hline $\mathrm{C} 5$ & $0.74766(13)$ & $-0.0969(5)$ & $0.87051(13)$ & $0.0451(6)$ \\
\hline C6 & $0.76742(13)$ & $0.0955(5)$ & $0.82406(13)$ & $0.0451(6)$ \\
\hline H6 & 0.7329 & 0.1779 & 0.7901 & $0.054 *$ \\
\hline $\mathrm{C} 7$ & $0.83837(12)$ & $0.1670(5)$ & $0.82758(12)$ & $0.0404(6)$ \\
\hline $\mathrm{H7}$ & 0.8529 & 0.2953 & 0.7950 & $0.049 *$ \\
\hline $\mathrm{C} 8$ & $0.95660(12)$ & $-0.1570(5)$ & $0.75198(12)$ & $0.0377(5)$ \\
\hline H8A & 0.9761 & -0.2628 & 0.7156 & $0.057 *$ \\
\hline H8B & 0.9113 & -0.0739 & 0.7323 & $0.057 *$ \\
\hline $\mathrm{H} 8 \mathrm{C}$ & 0.9491 & -0.2806 & 0.7905 & $0.057 *$ \\
\hline C9 & 1.09125 (12) & $0.0424(4)$ & $0.68596(10)$ & $0.0335(5)$ \\
\hline H9A & 1.0756 & -0.1523 & 0.6792 & $0.040^{*}$ \\
\hline H9B & 1.1438 & 0.0434 & 0.6931 & $0.040 *$ \\
\hline C10 & $1.06587(12)$ & $0.2015(4)$ & $0.62062(11)$ & $0.0339(5)$ \\
\hline C11 & $1.00736(11)$ & $0.0656(4)$ & $0.77853(10)$ & $0.0307(5)$ \\
\hline $\mathrm{C} 12$ & $1.26800(13)$ & $1.0394(5)$ & $0.89591(13)$ & $0.0465(6)$ \\
\hline $\mathrm{H} 12 \mathrm{~A}$ & 1.3122 & 1.1275 & 0.8866 & $0.070 *$ \\
\hline H12B & 1.2765 & 0.9371 & 0.9401 & $0.070 *$ \\
\hline $\mathrm{H} 12 \mathrm{C}$ & 1.2318 & 1.1815 & 0.8991 & $0.070 *$ \\
\hline $\mathrm{C} 13$ & $1.06544(11)$ & $0.1519(4)$ & $0.75071(10)$ & $0.0302(5)$ \\
\hline $\mathrm{C} 14$ & $1.10167(11)$ & $0.3605(4)$ & $0.79639(10)$ & $0.0286(5)$ \\
\hline $\mathrm{C} 15$ & $1.16259(11)$ & $0.5171(4)$ & $0.79317(11)$ & $0.0321(5)$ \\
\hline H15 & 1.1895 & 0.4955 & 0.7555 & $0.039 *$ \\
\hline $\mathrm{C} 16$ & $1.18318(11)$ & 0.7055 & $0.84620(11)$ & $0.0325(5)$ \\
\hline $\mathrm{C} 17$ & $1.14376(11)$ & $0.7377(4)$ & $0.90166(11)$ & $0.0332(5)$ \\
\hline H17 & 1.1586 & 0.8689 & 0.9373 & $0.040 *$ \\
\hline $\mathrm{C} 18$ & $1.08335(11)$ & $0.5814(4)$ & $0.90559(11)$ & $0.0332(5)$ \\
\hline H18 & 1.0564 & 0.6038 & 0.9432 & $0.040^{*}$ \\
\hline C19 & $1.06351(11)$ & 0.3918 (4) & $0.85293(10)$ & $0.0289(5)$ \\
\hline
\end{tabular}


supporting information

\begin{tabular}{lllll}
$\mathrm{C} 20$ & $1.07509(17)$ & $0.2359(7)$ & $0.50019(13)$ & $0.0614(8)$ \\
$\mathrm{H} 20 \mathrm{~A}$ & 1.0996 & 0.1470 & 0.4646 & $0.092^{*}$ \\
$\mathrm{H} 20 \mathrm{~B}$ & 1.0890 & 0.4311 & 0.5045 & $0.092^{*}$ \\
$\mathrm{H} 20 \mathrm{C}$ & 1.0237 & 0.2227 & 0.4865 & $0.092^{*}$ \\
\hline
\end{tabular}

Atomic displacement parameters $\left(\AA^{2}\right)$

\begin{tabular}{lllllll}
\hline & $U^{11}$ & $U^{22}$ & $U^{33}$ & $U^{12}$ & $U^{13}$ & $U^{23}$ \\
\hline C11 & $0.0484(4)$ & $0.0997(6)$ & $0.0658(5)$ & $-0.0277(4)$ & $0.0158(3)$ & $-0.0150(4)$ \\
O1 & $0.0611(11)$ & $0.0762(13)$ & $0.0302(10)$ & $-0.0255(10)$ & $0.0156(8)$ & $-0.0075(9)$ \\
O2 & $0.0426(10)$ & $0.0463(10)$ & $0.0448(10)$ & $-0.0091(7)$ & $0.0166(8)$ & $-0.0041(7)$ \\
O3 & $0.0578(11)$ & $0.0550(10)$ & $0.0263(8)$ & $0.0105(8)$ & $0.0117(7)$ & $-0.0011(7)$ \\
O4 & $0.0880(14)$ & $0.0593(12)$ & $0.0367(10)$ & $0.0352(11)$ & $0.0081(9)$ & $0.0053(8)$ \\
N1 & $0.0325(10)$ & $0.0326(10)$ & $0.0253(9)$ & $0.0025(7)$ & $0.0065(7)$ & $0.0004(7)$ \\
C1 & $0.0416(13)$ & $0.0373(12)$ & $0.0291(12)$ & $-0.0008(10)$ & $0.0097(10)$ & $0.0004(9)$ \\
C2 & $0.0394(13)$ & $0.0363(12)$ & $0.0324(12)$ & $-0.0015(10)$ & $0.0124(10)$ & $-0.0048(10)$ \\
C3 & $0.0472(14)$ & $0.0451(14)$ & $0.0381(13)$ & $-0.0045(11)$ & $0.0096(11)$ & $0.0023(11)$ \\
C4 & $0.0604(17)$ & $0.0491(15)$ & $0.0429(15)$ & $-0.0159(12)$ & $0.0181(13)$ & $-0.0009(12)$ \\
C5 & $0.0420(14)$ & $0.0543(15)$ & $0.0408(14)$ & $-0.0127(11)$ & $0.0123(11)$ & $-0.0147(12)$ \\
C6 & $0.0422(14)$ & $0.0513(15)$ & $0.0414(14)$ & $0.0002(11)$ & $0.0043(11)$ & $-0.0049(11)$ \\
C7 & $0.0424(14)$ & $0.0411(13)$ & $0.0392(14)$ & $-0.0013(10)$ & $0.0109(11)$ & $0.0019(10)$ \\
C8 & $0.0403(13)$ & $0.0369(12)$ & $0.0358(13)$ & $0.0031(10)$ & $0.0046(10)$ & $-0.0046(10)$ \\
C9 & $0.0419(13)$ & $0.0329(12)$ & $0.0265(11)$ & $0.0082(9)$ & $0.0075(9)$ & $-0.0016(9)$ \\
C10 & $0.0385(12)$ & $0.0353(13)$ & $0.0286(12)$ & $0.0004(10)$ & $0.0067(10)$ & $-0.0044(9)$ \\
C11 & $0.0369(12)$ & $0.0286(11)$ & $0.0261(11)$ & $0.0074(9)$ & $0.0028(9)$ & $0.0009(8)$ \\
C12 & $0.0437(14)$ & $0.0465(14)$ & $0.0492(15)$ & $-0.0077(11)$ & $0.0063(12)$ & $-0.0006(12)$ \\
C13 & $0.0385(12)$ & $0.0286(11)$ & $0.0234(11)$ & $0.0089(9)$ & $0.0035(9)$ & $0.0019(8)$ \\
C14 & $0.0335(12)$ & $0.0297(11)$ & $0.0231(11)$ & $0.0083(9)$ & $0.0054(9)$ & $0.0042(8)$ \\
C15 & $0.0363(12)$ & $0.0338(12)$ & $0.0284(12)$ & $0.0093(9)$ & $0.0124(9)$ & $0.0041(9)$ \\
C16 & $0.0340(12)$ & $0.0313(12)$ & $0.0332(12)$ & $0.0033(9)$ & $0.0078(9)$ & $0.0065(9)$ \\
C17 & $0.0394(12)$ & $0.0317(11)$ & $0.0291(12)$ & $0.0008(9)$ & $0.0063(10)$ & $-0.0016(9)$ \\
C18 & $0.0380(12)$ & $0.0361(12)$ & $0.0272(11)$ & $0.0026(9)$ & $0.0104(9)$ & $-0.0012(9)$ \\
C19 & $0.0318(11)$ & $0.0293(11)$ & $0.0260(11)$ & $0.0039(8)$ & $0.0054(9)$ & $0.0028(9)$ \\
C20 & $0.078(2)$ & $0.081(2)$ & $0.0256(13)$ & $0.0024(16)$ & $0.0099(13)$ & $0.0064(13)$ \\
& & & & & & \\
\hline & & & & & &
\end{tabular}

Geometric parameters $\left(\hat{A},{ }^{o}\right)$

\begin{tabular}{llll}
\hline $\mathrm{C} 11-\mathrm{C} 5$ & $1.746(2)$ & $\mathrm{C} 8-\mathrm{H} 8 \mathrm{~B}$ & 0.9800 \\
$\mathrm{O} 1-\mathrm{C} 1$ & $1.214(3)$ & $\mathrm{C} 8-\mathrm{H} 8 \mathrm{C}$ & 0.9800 \\
$\mathrm{O} 2-\mathrm{C} 16$ & $1.376(3)$ & $\mathrm{C} 9-\mathrm{C} 13$ & $1.500(3)$ \\
$\mathrm{O} 2-\mathrm{C} 12$ & $1.425(3)$ & $\mathrm{C} 9-\mathrm{C} 10$ & $1.501(3)$ \\
$\mathrm{O} 3-\mathrm{C} 10$ & $1.335(3)$ & $\mathrm{C} 9-\mathrm{H} 9 \mathrm{~A}$ & 0.9900 \\
$\mathrm{O} 3-\mathrm{C} 20$ & $1.445(3)$ & $\mathrm{C} 9-\mathrm{H} 9 \mathrm{~B}$ & 0.9900 \\
$\mathrm{O} 4-\mathrm{C} 10$ & $1.196(3)$ & $\mathrm{C} 11-\mathrm{C} 13$ & $1.357(3)$ \\
$\mathrm{N} 1-\mathrm{C} 1$ & $1.388(3)$ & $\mathrm{C} 12-\mathrm{H} 12 \mathrm{~A}$ & 0.9800 \\
$\mathrm{~N} 1-\mathrm{C} 19$ & $1.420(3)$ & $\mathrm{C} 12-\mathrm{H} 12 \mathrm{~B}$ & 0.9800 \\
$\mathrm{~N} 1-\mathrm{C} 11$ & $1.424(3)$ & $\mathrm{C} 12-\mathrm{H} 12 \mathrm{C}$ & 0.9800 \\
$\mathrm{C} 1-\mathrm{C} 2$ & $1.496(3)$ & $\mathrm{C} 13-\mathrm{C} 14$ & $1.451(3)$
\end{tabular}




\begin{tabular}{|c|c|c|c|}
\hline $\mathrm{C} 2-\mathrm{C} 7$ & $1.381(3)$ & $\mathrm{C} 14-\mathrm{C} 15$ & $1.393(3)$ \\
\hline $\mathrm{C} 2-\mathrm{C} 3$ & $1.390(3)$ & $\mathrm{C} 14-\mathrm{C} 19$ & $1.398(3)$ \\
\hline $\mathrm{C} 3-\mathrm{C} 4$ & $1.384(3)$ & $\mathrm{C} 15-\mathrm{C} 16$ & $1.386(3)$ \\
\hline $\mathrm{C} 3-\mathrm{H} 3$ & 0.9500 & $\mathrm{C} 15-\mathrm{H} 15$ & 0.9500 \\
\hline $\mathrm{C} 4-\mathrm{C} 5$ & $1.376(4)$ & $\mathrm{C} 16-\mathrm{C} 17$ & $1.396(3)$ \\
\hline $\mathrm{C} 4-\mathrm{H} 4$ & 0.9500 & $\mathrm{C} 17-\mathrm{C} 18$ & $1.386(3)$ \\
\hline $\mathrm{C} 5-\mathrm{C} 6$ & $1.379(4)$ & $\mathrm{C} 17-\mathrm{H} 17$ & 0.9500 \\
\hline $\mathrm{C} 6-\mathrm{C} 7$ & $1.386(3)$ & $\mathrm{C} 18-\mathrm{C} 19$ & $1.383(3)$ \\
\hline $\mathrm{C} 6-\mathrm{H} 6$ & 0.9500 & $\mathrm{C} 18-\mathrm{H} 18$ & 0.9500 \\
\hline $\mathrm{C} 7-\mathrm{H} 7$ & 0.9500 & $\mathrm{C} 20-\mathrm{H} 20 \mathrm{~A}$ & 0.9800 \\
\hline $\mathrm{C} 8-\mathrm{C} 11$ & $1.490(3)$ & $\mathrm{C} 20-\mathrm{H} 20 \mathrm{~B}$ & 0.9800 \\
\hline $\mathrm{C} 8-\mathrm{H} 8 \mathrm{~A}$ & 0.9800 & $\mathrm{C} 20-\mathrm{H} 20 \mathrm{C}$ & 0.9800 \\
\hline $\mathrm{C} 16-\mathrm{O} 2-\mathrm{C} 12$ & $117.11(17)$ & $\mathrm{O} 4-\mathrm{C} 10-\mathrm{C} 9$ & $126.3(2)$ \\
\hline $\mathrm{C} 10-\mathrm{O} 3-\mathrm{C} 20$ & $116.21(19)$ & $\mathrm{O} 3-\mathrm{C} 10-\mathrm{C} 9$ & $110.39(18)$ \\
\hline $\mathrm{C} 1-\mathrm{N} 1-\mathrm{C} 19$ & $121.17(17)$ & $\mathrm{C} 13-\mathrm{C} 11-\mathrm{N} 1$ & $108.76(18)$ \\
\hline $\mathrm{C} 1-\mathrm{N} 1-\mathrm{C} 11$ & $129.92(18)$ & $\mathrm{C} 13-\mathrm{C} 11-\mathrm{C} 8$ & $127.59(19)$ \\
\hline $\mathrm{C} 19-\mathrm{N} 1-\mathrm{C} 11$ & $107.69(16)$ & $\mathrm{N} 1-\mathrm{C} 11-\mathrm{C} 8$ & $123.55(18)$ \\
\hline $\mathrm{O} 1-\mathrm{C} 1-\mathrm{N} 1$ & $120.0(2)$ & $\mathrm{O} 2-\mathrm{C} 12-\mathrm{H} 12 \mathrm{~A}$ & 109.5 \\
\hline $\mathrm{O} 1-\mathrm{C} 1-\mathrm{C} 2$ & $118.03(19)$ & $\mathrm{O} 2-\mathrm{C} 12-\mathrm{H} 12 \mathrm{~B}$ & 109.5 \\
\hline $\mathrm{N} 1-\mathrm{C} 1-\mathrm{C} 2$ & $121.79(19)$ & $\mathrm{H} 12 \mathrm{~A}-\mathrm{C} 12-\mathrm{H} 12 \mathrm{~B}$ & 109.5 \\
\hline $\mathrm{C} 7-\mathrm{C} 2-\mathrm{C} 3$ & $119.8(2)$ & $\mathrm{O} 2-\mathrm{C} 12-\mathrm{H} 12 \mathrm{C}$ & 109.5 \\
\hline $\mathrm{C} 7-\mathrm{C} 2-\mathrm{C} 1$ & $122.0(2)$ & $\mathrm{H} 12 \mathrm{~A}-\mathrm{C} 12-\mathrm{H} 12 \mathrm{C}$ & 109.5 \\
\hline $\mathrm{C} 3-\mathrm{C} 2-\mathrm{C} 1$ & $117.2(2)$ & $\mathrm{H} 12 \mathrm{~B}-\mathrm{C} 12-\mathrm{H} 12 \mathrm{C}$ & 109.5 \\
\hline $\mathrm{C} 4-\mathrm{C} 3-\mathrm{C} 2$ & $120.0(2)$ & $\mathrm{C} 11-\mathrm{C} 13-\mathrm{C} 14$ & $108.55(18)$ \\
\hline $\mathrm{C} 4-\mathrm{C} 3-\mathrm{H} 3$ & 120.0 & $\mathrm{C} 11-\mathrm{C} 13-\mathrm{C} 9$ & $126.6(2)$ \\
\hline $\mathrm{C} 2-\mathrm{C} 3-\mathrm{H} 3$ & 120.0 & $\mathrm{C} 14-\mathrm{C} 13-\mathrm{C} 9$ & $124.73(19)$ \\
\hline $\mathrm{C} 5-\mathrm{C} 4-\mathrm{C} 3$ & $119.2(2)$ & $\mathrm{C} 15-\mathrm{C} 14-\mathrm{C} 19$ & $119.89(19)$ \\
\hline $\mathrm{C} 5-\mathrm{C} 4-\mathrm{H} 4$ & 120.4 & $\mathrm{C} 15-\mathrm{C} 14-\mathrm{C} 13$ & $132.90(19)$ \\
\hline $\mathrm{C} 3-\mathrm{C} 4-\mathrm{H} 4$ & 120.4 & $\mathrm{C} 19-\mathrm{C} 14-\mathrm{C} 13$ & $107.21(18)$ \\
\hline $\mathrm{C} 4-\mathrm{C} 5-\mathrm{C} 6$ & $121.5(2)$ & $\mathrm{C} 16-\mathrm{C} 15-\mathrm{C} 14$ & $118.56(18)$ \\
\hline $\mathrm{C} 4-\mathrm{C} 5-\mathrm{Cl1}$ & $119.7(2)$ & $\mathrm{C} 16-\mathrm{C} 15-\mathrm{H} 15$ & 120.7 \\
\hline $\mathrm{C} 6-\mathrm{C} 5-\mathrm{Cl} 1$ & $118.8(2)$ & $\mathrm{C} 14-\mathrm{C} 15-\mathrm{H} 15$ & 120.7 \\
\hline $\mathrm{C} 5-\mathrm{C} 6-\mathrm{C} 7$ & $119.0(2)$ & $\mathrm{O} 2-\mathrm{C} 16-\mathrm{C} 15$ & $116.01(18)$ \\
\hline $\mathrm{C} 5-\mathrm{C} 6-\mathrm{H} 6$ & 120.5 & $\mathrm{O} 2-\mathrm{C} 16-\mathrm{C} 17$ & $123.2(2)$ \\
\hline $\mathrm{C} 7-\mathrm{C} 6-\mathrm{H} 6$ & 120.5 & $\mathrm{C} 15-\mathrm{C} 16-\mathrm{C} 17$ & $120.8(2)$ \\
\hline $\mathrm{C} 2-\mathrm{C} 7-\mathrm{C} 6$ & $120.3(2)$ & $\mathrm{C} 18-\mathrm{C} 17-\mathrm{C} 16$ & $121.1(2)$ \\
\hline $\mathrm{C} 2-\mathrm{C} 7-\mathrm{H} 7$ & 119.8 & $\mathrm{C} 18-\mathrm{C} 17-\mathrm{H} 17$ & 119.5 \\
\hline $\mathrm{C} 6-\mathrm{C} 7-\mathrm{H} 7$ & 119.8 & $\mathrm{C} 16-\mathrm{C} 17-\mathrm{H} 17$ & 119.5 \\
\hline $\mathrm{C} 11-\mathrm{C} 8-\mathrm{H} 8 \mathrm{~A}$ & 109.5 & $\mathrm{C} 19-\mathrm{C} 18-\mathrm{C} 17$ & $117.84(19)$ \\
\hline $\mathrm{C} 11-\mathrm{C} 8-\mathrm{H} 8 \mathrm{~B}$ & 109.5 & $\mathrm{C} 19-\mathrm{C} 18-\mathrm{H} 18$ & 121.1 \\
\hline $\mathrm{H} 8 \mathrm{~A}-\mathrm{C} 8-\mathrm{H} 8 \mathrm{~B}$ & 109.5 & $\mathrm{C} 17-\mathrm{C} 18-\mathrm{H} 18$ & 121.1 \\
\hline $\mathrm{C} 11-\mathrm{C} 8-\mathrm{H} 8 \mathrm{C}$ & 109.5 & $\mathrm{C} 18-\mathrm{C} 19-\mathrm{C} 14$ & $121.80(19)$ \\
\hline $\mathrm{H} 8 \mathrm{~A}-\mathrm{C} 8-\mathrm{H} 8 \mathrm{C}$ & 109.5 & $\mathrm{C} 18-\mathrm{C} 19-\mathrm{N} 1$ & $130.35(19)$ \\
\hline $\mathrm{H} 8 \mathrm{~B}-\mathrm{C} 8-\mathrm{H} 8 \mathrm{C}$ & 109.5 & $\mathrm{C} 14-\mathrm{C} 19-\mathrm{N} 1$ & $107.77(17)$ \\
\hline $\mathrm{C} 13-\mathrm{C} 9-\mathrm{C} 10$ & $114.56(17)$ & $\mathrm{O} 3-\mathrm{C} 20-\mathrm{H} 20 \mathrm{~A}$ & 109.5 \\
\hline $\mathrm{C} 13-\mathrm{C} 9-\mathrm{H} 9 \mathrm{~A}$ & 108.6 & $\mathrm{O} 3-\mathrm{C} 20-\mathrm{H} 20 \mathrm{~B}$ & 109.5 \\
\hline $\mathrm{C} 10-\mathrm{C} 9-\mathrm{H} 9 \mathrm{~A}$ & 108.6 & $\mathrm{H} 20 \mathrm{~A}-\mathrm{C} 20-\mathrm{H} 20 \mathrm{~B}$ & 109.5 \\
\hline
\end{tabular}




\begin{tabular}{|c|c|c|c|}
\hline $\mathrm{C} 13-\mathrm{C} 9-\mathrm{H} 9 \mathrm{~B}$ & 108.6 & $\mathrm{O} 3-\mathrm{C} 20-\mathrm{H} 20 \mathrm{C}$ & 109.5 \\
\hline $\mathrm{C} 10-\mathrm{C} 9-\mathrm{H} 9 \mathrm{~B}$ & 108.6 & $\mathrm{H} 20 \mathrm{~A}-\mathrm{C} 20-\mathrm{H} 20 \mathrm{C}$ & 109.5 \\
\hline $\mathrm{H} 9 \mathrm{~A}-\mathrm{C} 9-\mathrm{H} 9 \mathrm{~B}$ & 107.6 & $\mathrm{H} 20 \mathrm{~B}-\mathrm{C} 20-\mathrm{H} 20 \mathrm{C}$ & 109.5 \\
\hline $\mathrm{O} 4-\mathrm{C} 10-\mathrm{O} 3$ & $123.3(2)$ & & \\
\hline $\mathrm{C} 19-\mathrm{N} 1-\mathrm{C} 1-\mathrm{O} 1$ & $-13.8(3)$ & $\mathrm{N} 1-\mathrm{C} 11-\mathrm{C} 13-\mathrm{C} 9$ & $176.99(18)$ \\
\hline $\mathrm{C} 11-\mathrm{N} 1-\mathrm{C} 1-\mathrm{O} 1$ & $152.0(2)$ & $\mathrm{C} 8-\mathrm{C} 11-\mathrm{C} 13-\mathrm{C} 9$ & $0.6(3)$ \\
\hline $\mathrm{C} 19-\mathrm{N} 1-\mathrm{C} 1-\mathrm{C} 2$ & $161.21(19)$ & $\mathrm{C} 10-\mathrm{C} 9-\mathrm{C} 13-\mathrm{C} 11$ & $94.3(3)$ \\
\hline $\mathrm{C} 11-\mathrm{N} 1-\mathrm{C} 1-\mathrm{C} 2$ & $-33.0(3)$ & $\mathrm{C} 10-\mathrm{C} 9-\mathrm{C} 13-\mathrm{C} 14$ & $-89.3(2)$ \\
\hline $\mathrm{O} 1-\mathrm{C} 1-\mathrm{C} 2-\mathrm{C} 7$ & $123.7(2)$ & $\mathrm{C} 11-\mathrm{C} 13-\mathrm{C} 14-\mathrm{C} 15$ & $-179.5(2)$ \\
\hline $\mathrm{N} 1-\mathrm{C} 1-\mathrm{C} 2-\mathrm{C} 7$ & $-51.4(3)$ & $\mathrm{C} 9-\mathrm{C} 13-\mathrm{C} 14-\mathrm{C} 15$ & $3.5(3)$ \\
\hline $\mathrm{O} 1-\mathrm{C} 1-\mathrm{C} 2-\mathrm{C} 3$ & $-44.9(3)$ & $\mathrm{C} 11-\mathrm{C} 13-\mathrm{C} 14-\mathrm{C} 19$ & $0.9(2)$ \\
\hline $\mathrm{N} 1-\mathrm{C} 1-\mathrm{C} 2-\mathrm{C} 3$ & $140.0(2)$ & $\mathrm{C} 9-\mathrm{C} 13-\mathrm{C} 14-\mathrm{C} 19$ & $-176.05(18)$ \\
\hline $\mathrm{C} 7-\mathrm{C} 2-\mathrm{C} 3-\mathrm{C} 4$ & $0.1(3)$ & $\mathrm{C} 19-\mathrm{C} 14-\mathrm{C} 15-\mathrm{C} 16$ & $-1.1(3)$ \\
\hline $\mathrm{C} 1-\mathrm{C} 2-\mathrm{C} 3-\mathrm{C} 4$ & $169.0(2)$ & $\mathrm{C} 13-\mathrm{C} 14-\mathrm{C} 15-\mathrm{C} 16$ & $179.3(2)$ \\
\hline $\mathrm{C} 2-\mathrm{C} 3-\mathrm{C} 4-\mathrm{C} 5$ & $-2.0(4)$ & $\mathrm{C} 12-\mathrm{O} 2-\mathrm{C} 16-\mathrm{C} 15$ & $-177.32(19)$ \\
\hline $\mathrm{C} 3-\mathrm{C} 4-\mathrm{C} 5-\mathrm{C} 6$ & $1.9(4)$ & $\mathrm{C} 12-\mathrm{O} 2-\mathrm{C} 16-\mathrm{C} 17$ & $2.3(3)$ \\
\hline $\mathrm{C} 3-\mathrm{C} 4-\mathrm{C} 5-\mathrm{Cl} 1$ & $-176.15(19)$ & $\mathrm{C} 14-\mathrm{C} 15-\mathrm{C} 16-\mathrm{O} 2$ & $179.62(18)$ \\
\hline $\mathrm{C} 4-\mathrm{C} 5-\mathrm{C} 6-\mathrm{C} 7$ & $0.0(4)$ & $\mathrm{C} 14-\mathrm{C} 15-\mathrm{C} 16-\mathrm{C} 17$ & $0.0(3)$ \\
\hline $\mathrm{C} 11-\mathrm{C} 5-\mathrm{C} 6-\mathrm{C} 7$ & $178.05(18)$ & $\mathrm{O} 2-\mathrm{C} 16-\mathrm{C} 17-\mathrm{C} 18$ & $-179.13(19)$ \\
\hline $\mathrm{C} 3-\mathrm{C} 2-\mathrm{C} 7-\mathrm{C} 6$ & $1.8(3)$ & $\mathrm{C} 15-\mathrm{C} 16-\mathrm{C} 17-\mathrm{C} 18$ & $0.5(3)$ \\
\hline $\mathrm{C} 1-\mathrm{C} 2-\mathrm{C} 7-\mathrm{C} 6$ & $-166.5(2)$ & $\mathrm{C} 16-\mathrm{C} 17-\mathrm{C} 18-\mathrm{C} 19$ & $0.2(3)$ \\
\hline $\mathrm{C} 5-\mathrm{C} 6-\mathrm{C} 7-\mathrm{C} 2$ & $-1.8(4)$ & $\mathrm{C} 17-\mathrm{C} 18-\mathrm{C} 19-\mathrm{C} 14$ & $-1.4(3)$ \\
\hline $\mathrm{C} 20-\mathrm{O} 3-\mathrm{C} 10-\mathrm{O} 4$ & $0.6(3)$ & $\mathrm{C} 17-\mathrm{C} 18-\mathrm{C} 19-\mathrm{N} 1$ & $-177.6(2)$ \\
\hline $\mathrm{C} 20-\mathrm{O} 3-\mathrm{C} 10-\mathrm{C} 9$ & $-179.1(2)$ & $\mathrm{C} 15-\mathrm{C} 14-\mathrm{C} 19-\mathrm{C} 18$ & $1.9(3)$ \\
\hline $\mathrm{C} 13-\mathrm{C} 9-\mathrm{C} 10-\mathrm{O} 4$ & $-2.9(3)$ & $\mathrm{C} 13-\mathrm{C} 14-\mathrm{C} 19-\mathrm{C} 18$ & $-178.48(19)$ \\
\hline $\mathrm{C} 13-\mathrm{C} 9-\mathrm{C} 10-\mathrm{O} 3$ & $176.79(19)$ & $\mathrm{C} 15-\mathrm{C} 14-\mathrm{C} 19-\mathrm{N} 1$ & $178.81(17)$ \\
\hline $\mathrm{C} 1-\mathrm{N} 1-\mathrm{C} 11-\mathrm{C} 13$ & $-168.3(2)$ & $\mathrm{C} 13-\mathrm{C} 14-\mathrm{C} 19-\mathrm{N} 1$ & $-1.5(2)$ \\
\hline $\mathrm{C} 19-\mathrm{N} 1-\mathrm{C} 11-\mathrm{C} 13$ & $-1.1(2)$ & $\mathrm{C} 1-\mathrm{N} 1-\mathrm{C} 19-\mathrm{C} 18$ & $-13.2(3)$ \\
\hline $\mathrm{C} 1-\mathrm{N} 1-\mathrm{C} 11-\mathrm{C} 8$ & $8.3(3)$ & $\mathrm{C} 11-\mathrm{N} 1-\mathrm{C} 19-\mathrm{C} 18$ & $178.2(2)$ \\
\hline $\mathrm{C} 19-\mathrm{N} 1-\mathrm{C} 11-\mathrm{C} 8$ & $175.50(19)$ & $\mathrm{C} 1-\mathrm{N} 1-\mathrm{C} 19-\mathrm{C} 14$ & $170.21(18)$ \\
\hline $\mathrm{N} 1-\mathrm{C} 11-\mathrm{C} 13-\mathrm{C} 14$ & $0.1(2)$ & $\mathrm{C} 11-\mathrm{N} 1-\mathrm{C} 19-\mathrm{C} 14$ & $1.6(2)$ \\
\hline $\mathrm{C} 8-\mathrm{C} 11-\mathrm{C} 13-\mathrm{C} 14$ & $-176.28(19)$ & & \\
\hline
\end{tabular}

Hydrogen-bond geometry $\left(\AA,{ }^{\circ}\right)$

\begin{tabular}{lllll}
\hline$D-\mathrm{H} \cdots A$ & $D-\mathrm{H}$ & $\mathrm{H} \cdots A$ & $D \cdots A$ & $D-\mathrm{H} \cdots A$ \\
\hline $\mathrm{C} 18-\mathrm{H} 18 \cdots \mathrm{O} 1^{\mathrm{i}}$ & 0.95 & 2.34 & $3.260(3)$ & 162 \\
$\mathrm{C} 3-\mathrm{H} 3 \cdots \mathrm{O} 1^{\mathrm{ii}}$ & 0.95 & 2.59 & $3.480(3)$ & 156 \\
$\mathrm{C} 9-\mathrm{H} 9 A \cdots \mathrm{O} 4^{\mathrm{iii}}$ & 0.99 & 2.62 & $3.565(3)$ & 160 \\
$\mathrm{C} 15-\mathrm{H} 15 \cdots \mathrm{O} 2^{\mathrm{iv}}$ & 0.95 & 2.48 & $3.419(2)$ & 170 \\
\hline
\end{tabular}

Symmetry codes: (i) $-x+2,-y+1,-z+2$; (ii) $-x+2,-y,-z+2$; (iii) $x, y-1, z$; (iv) $-x+5 / 2, y-1 / 2,-z+3 / 2$. 